\title{
Challenges Involved in Incorporating Personalised Treatment Plan as Routine Care of Patients with Diabetes
}

This article was published in the following Dove Press journal: Pharmacogenomics and Personalized Medicine

\author{
Ranjit Unnikrishnan' \\ Venkatesan Radha ${ }^{2}$ \\ Viswanathan Mohan (D) \\ 'Department of Diabetology, Dr. Mohan's \\ Diabetes Specialities Centre and Madras \\ Diabetes Research Foundation, Chennai, \\ India; ${ }^{2}$ Department of Molecular \\ Genetics, Madras Diabetes Research \\ Foundation, Chennai, India
}

\begin{abstract}
Diabetes is a heterogenous disorder, and patients with this disorder vary considerably in their clinical presentation, response to therapy and risk of complications. Expanding knowledge about the pathophysiology of various forms of diabetes has raised the possibility that diagnostic and therapeutic modalities can be tailored to the individual patient in a personalized manner. The recent publication of a Consensus Statement on precision diabetes care underlines the major strides made in this field in the recent past. However, while personalized diabetes care has the potential to significantly improve outcomes in patients with diabetes in a safe and cost-effective manner, its wider application presents several challenges, especially in resource-strained settings. These challenges pertain equally to precision diagnostics, precision therapeutics and precision monitoring. This article discusses some of the important challenges that care providers are likely to face in applying the personalized approach in caring for their patients with diabetes, in the context of diagnosis and management of type 1 diabetes, type 2 diabetes and monogenic forms of diabetes. Suggestions are also presented for overcoming some of these challenges.
\end{abstract}

Keywords: type 1 diabetes, type 2 diabetes, monogenic forms of diabetes, personalised treatment

\section{Introduction}

The burgeoning diabetes pandemic has placed considerable strain on the health care systems of countries worldwide, and is associated with considerable morbidity and mortality pertaining to its effects on various organs and systems. In consonance with the exploding prevalence of diabetes, research has also progressed apace into avenues for its management, with several classes of therapeutic agents having been made available over the past five decades. However, diabetes control remains suboptimal in many parts of the world, placing individuals at risk of developing acute and chronic complications.

It has long been recognized that diabetes is not a homogenous disease. The ancient Indian texts of Charaka and Sushruta provide descriptions of two distinct forms of diabetes that correspond to type 1 (T1D) and type diabetes (T2D) and recommend different forms of treatment for both- perhaps the first record of precision diabetes care. ${ }^{1}$ In the modern era, studies by Himsworth showed that individuals with diabetes could be subdivided into insulin-sensitive and insulinresistant types, with important implications for treatment. ${ }^{2}$
Correspondence: Viswanathan Mohan

Dr. Mohan's Diabetes Specialities Centre,

Care \& Madras Diabetes Research

Foundation, ICMR Centre for Advanced

Research on Diabetes No 4, Conran

Smith Road, Gopalapuram, Chennai, 600

086, India

Tel +9|4443968888

Fax +914428350935

Email drmohans@diabetes.ind.in
Pharmacogenomics and Personalized Medicine 2021:14 327-333

DovePres:

$f$

in 1 
Further development of the concept of personalized or precision diabetes care led the American Diabetes Association (ADA) to launch a Precision Medicine in Diabetes Initiative (PMDI). The first Consensus Report of this initiative has recently been published. ${ }^{3}$

\section{Methodology}

For the purpose of preparing this narrative review, we searched PubMed for articles in English, published between 2000 and 2020, using the keywords "diabetes", "precision diabetes" and "personalized diabetes".

\section{Precision Medicine vs Personalized Medicine}

The term "precision medicine" refers to the utilization of clinical, biochemical, anthropometric and genetic data to determine the best possible management plan for a patient. When the application of precision medicine is modified by patient factors such as beliefs and preferences, affordability and accessibility, the term "personalized medicine" is used. $^{4}$

Application of the concept of personalized medicine has the potential to significantly improve outcomes in patient care in diabetes, by improving glycemic control and preventing complications with the lowest possible risk of side-effects of treatment. The precision approach can be applied to the prevention, diagnosis, monitoring and management of different types of diabetes.

\section{Challenges in Application of Personalised Medicine in Diabetes Care}

While personalized medicine has the potential to be a game-changer in the way we treat diabetes, its application is not without major challenges, not only in developing countries where the majority of people with diabetes live, but also in advanced nations. The PMDI Consensus Report has spelt out these challenges in detail and these are listed below (Table 1).

1. Challenges in precision diagnostics and classification

2. Challenges in precision prevention

3. Challenges in precision management (monitoring and treatment)
Table I Summary of Challenges in Precision Diabetes

\begin{tabular}{|c|c|}
\hline $\begin{array}{l}\text { Challenges in } \\
\text { diagnosis and } \\
\text { classification }\end{array}$ & $\begin{array}{l}\text { - Many of the diagnostic tests not easily available } \\
\text { - (e.g. GAD antibody assay) } \\
\text { - Many of the biomarkers used in differentiating } \\
\text { - Most of the approaches used for clustering } \\
\text { require variables to be continuously distribu- } \\
\text { ted; this adversely affects accuracy }\end{array}$ \\
\hline $\begin{array}{l}\text { Challenges in } \\
\text { prevention }\end{array}$ & $\begin{array}{l}\text { - Appropriate technologies often not available } \\
\text { (e.g. apps) } \\
\text { - Patient motivation remains a challenge }\end{array}$ \\
\hline $\begin{array}{l}\text { Challenges in } \\
\text { monitoring and } \\
\text { treatment }\end{array}$ & $\begin{array}{l}\text { - Insufficient data on drug response in non- } \\
\text { white populations (who constitute the bulk of } \\
\text { - Reople with T2D) } \\
\text { - } \text { tional analysis is not easy to meet } \\
\text { - Few large real-world studies on the efficacy of } \\
\text { - Care providion approaches } \\
\text { mit complex information regarding precision } \\
\text { care to patients }\end{array}$ \\
\hline
\end{tabular}

Note: Data from Chung et al, 2020. ${ }^{3}$

Abbreviation: GAD, glutamic acid decarboxylase.

This article will focus on the challenges associated with personalized diabetes diagnosis (and classification) and treatment.

\section{Challenges in Diagnosis and Classification of Diabetes}

Type 2 diabetes is the most common form of diabetes worldwide and accounts for more than $90 \%$ of the disease burden due to diabetes. It has long been known, however, that $\mathrm{T} 2 \mathrm{D}$ is a heterogeneous disease that varies in its pathophysiology, clinical features, management options and susceptibility to complications. With this in mind, attempts have been made to identify subgroups or "clusters" of T2D with distinct pathophysiological and clinical features. Perhaps the most significant step in this direction has been the work of Ahlquist et al in the Scandinavian (Swedish and Finnish) population. ${ }^{5}$ Using a few biochemical, anthropometric and clinical variables, they divided individuals diagnosed with "type 2 diabetes" into five distinct clusters and described the clinical characteristics of each cluster. These clusters have been named as follows: Severe Autoimmune Diabetes (SAID), Severe Insulin Dependent Diabetes (SIDD), Severe Insulin Resistant Diabetes (SIRD), mild obesity-related diabetes (MOD) and mild age-related diabetes (MARD). They 
suggest that individuals in the SAID cluster represent T1D hitherto misdiagnosed as T2D, since these individuals have evidence of beta-cell autoimmunity. Individuals in the SIDD and SIRD groups have worse hyperglycemia and are more prone to diabetes complications, and differ in the most ideal treatment options (insulin for SIDD, insulin sensitisers for SIRD). MARD and MOD are relatively mild forms of T2D with low risk of complications.

The publication of the above paper has raised hopes worldwide over the feasibility of a precision approach to the management of T2D. However, attempts to replicate these clusters in other populations have shown mixed results, with excellent replication in some populations and incomplete replication in others. ${ }^{6-9}$ For instance, the attempt from India showed that while the SIDD and MARD clusters could be distinguished readily in this population, those diagnosed as SIRD and MOD behaved differently from those described in the Scandinavian population (Table 2). Also, the Indian study suggested the possibility of two novel clusters termed as combined insulin resistance and deficient diabetes (CIRDD) and insulin-resistant obese diabetes (IROD) with features distinct from any cluster identified in the Scandinavian population. These findings raise the possibility that identification of subgroups of T2D will have to be performed separately for different ethnic groups, to find out the most relevant clusters for each population.
Another challenge in clustering arises from the use of different biochemical variables in the model, many of which are not routinely performed in patients as a part of clinical care. For instance, the Scandinavian clustering utilized variables such as glutamic acid decarboxylase (GAD) antibodies, C-peptide assay and homeostasis assessment of beta-cell function and insulin resistance, none of which are routinely assessed in patients with T2D outside a research study. The problem is particularly relevant for developing countries, where these investigations are neither readily available nor affordable for the vast majority of patients. With this in mind, the Indian attempt at identifying clusters of T2D has restricted itself to using readily available variables such as age, body weight, waist circumference, serum lipids and glycated hemoglobin (HbA1c). Further studies are needed to evaluate the cost-effectiveness of this approach vis-à-vis the adoption of appropriate treatment and prevention of complications. There is now some evidence, particularly in European populations, that stratifying patients using simple continuous clinical variables such as age at diagnosis better predicts outcomes and progression of disease than a datadriven approach. ${ }^{10}$

Yet another challenge in the clustering approach is the fact that many of the variables utilized tend to change with time. The currently published literature on clustering pertains only to individuals with newly diagnosed or short-duration diabetes. It is not known whether these findings apply equally to those with longer durations of diabetes.

Table 2 Summary of "Clusters" of Type 2 Diabetes in Asian Indians

\begin{tabular}{|c|c|c|}
\hline Name of Cluster & Characteristics & Implications \\
\hline $\begin{array}{l}\text { SIDD (Severe insulin deficient } \\
\text { diabetes) }\end{array}$ & $\begin{array}{l}\text { - Early-onset diabetes } \\
\text { - Relatively low BMI \& waist circumference } \\
\text { - Low HOMA-B and HOMA-IR } \\
\text { - Low C-peptide levels } \\
\text { - High HbAlc }\end{array}$ & $\begin{array}{l}\text { - Increased risk for retinopathy } \\
\text { - May benefit from early insulin }\end{array}$ \\
\hline $\begin{array}{l}\text { IROD (Insulin resistant obese } \\
\text { diabetes) }\end{array}$ & $\begin{array}{l}\text { - High BMI \& waist circumference } \\
\text { - High C-peptide levels }\end{array}$ & $\begin{array}{l}\text { - Increased risk for diabetic kidney disease } \\
\text { - Likely to respond better to insulin sensitisers }\end{array}$ \\
\hline $\begin{array}{l}\text { CIRDD (Combined insulin resistant } \\
\text { and deficient diabetes) }\end{array}$ & $\begin{array}{l}\text { - BMI, waist circumference, HOMA-B and HOMA-IR } \\
\text { intermediate between SIDD \& IROD } \\
\text { - High triglycerides \& low HDL- Cholesterol }\end{array}$ & $\begin{array}{l}\text { - Increased risk for both retinopathy and dia- } \\
\text { betic kidney disease } \\
\text { - Likely to require a combination of insulin- } \\
\text { providing and insulin-sparing therapies }\end{array}$ \\
\hline MARD (Mild age-related diabetes) & $\begin{array}{l}\text { - Older than patients in other clusters } \\
\text { - Highest HDL -Cholesterol } \\
\text { - Fairly preserved C-peptide levels } \\
\text { - Milder diabetes }\end{array}$ & $\begin{array}{l}\text { - Low risk for complications } \\
\text { - May benefit from less aggressive treatment }\end{array}$ \\
\hline
\end{tabular}

Note: Data from Anjana et al, 2020. ${ }^{9}$

Abbreviations: BMI, body mass index; HOMA, homeostasis model assessment; HDL, high-density lipoprotein. 
Similar challenges underlie the personalized approach to the diagnosis of T1D. While T1D can readily be differentiated into antibody-positive (T1a) and antibody-negative (T1b) forms, it is still not known whether this distinction has any implications in the management or risk of complications related to T1D. Also, it has been shown that a significant proportion of Asian Indian individuals with T1D are negative for all the known pancreatic autoantibodies. ${ }^{11}$ It is not known whether this phenotype represents a distinct form of the disease, or whether these patients would test positive for other, hitherto unknown, autoantibodies. Therefore, the role of the precision approach in the diagnosis of T1D remains restricted at present.

Monogenic diabetes is considered the "poster child" of precision diabetes care, as making an accurate molecular diagnosis enables the institution of personalized diabetes management in many of these cases. Until recently, the diagnosis of monogenic diabetes (some forms of which continue to be described under the term "MODY"- maturity onset diabetes of the young) was based entirely on clinical criteria. ${ }^{12}$ However, it is now known that clinical criteria are neither sensitive or specific enough and for an accurate diagnosis, genetic testing is essential. This raises the issue of whom to be referred for genetic testing, a question that assumes particular importance in the context of developing countries, where these tests are not easily available or affordable. While all patients with young-onset diabetes who do not fall into an unequivocal diagnosis of T1D should ideally be tested for monogenic defects, such an approach is neither feasible nor cost-effective. Therefore, the use of the precision approach to the diagnosis of monogenic diabetes involves the identification of distinct phenotypes of young-onset diabetes who are most likely to i) to harbor a pathogenic mutation and ii) to benefit the most from the diagnosis of such a mutation.

The phenotypes in which a genetic diagnosis is likely to provide the greatest clinical benefit are: ${ }^{13}$

\section{Neonatal diabetes}

2. Familial diabetes with affected parent [conventionally termed MODY1 and MODY3, due to mutations in hepatocyte nuclear factor (HNF) $4 \mathrm{~A}$ and $1 \mathrm{~A}$, respectively]

3. Familial mild non-progressive fasting hyperglycemia [conventionally termed MODY2, due to mutations in the glucokinase (GCK) gene]

4. Diabetes with extrapancreatic manifestations (syndromic forms of diabetes)
Restricting genetic testing to individuals with these phenotypes is likely to provide the greatest diagnostic yield as well as improve cost-effectiveness.

Even after a mutation has been identified, it is important to decide whether the mutation is actually responsible for the clinical phenotype of the patient (ie pathogenic) and whether this information can be used to alter the treatment (ie actionable). The situation is further complicated by the presence of variants of unknown significance (VUS), which, in fact, are seen more frequently than the benign and pathogenic variants. Deciding whether a VUS is responsible for the patient's clinical presentation is one of the most challenging aspects of genetic diagnosis of diabetes. ${ }^{14}$ The use of large global databases like the gnomAD can help clarify the situation, but often requires a level of expertise that is not easily available in many

Table 3 Implications of Accurate Molecular Diagnosis on Common and Rare Types of MODY

\begin{tabular}{|c|c|c|}
\hline Subtype & $\begin{array}{l}\text { Gene } \\
\text { Involved }\end{array}$ & Management \\
\hline \multicolumn{3}{|c|}{ Common subtypes } \\
\hline MODY3 & HNFIA & \multirow{2}{*}{$\begin{array}{l}\text { Low dose sulfonylurea; may rarely require } \\
\text { insulin at later stages }\end{array}$} \\
\hline MODYI & HNF4A & \\
\hline MODY2 & GCK & No treatment required \\
\hline MODY5 & HNFIB & Usually require insulin \\
\hline \multicolumn{3}{|c|}{ Rare subtypes* } \\
\hline MODYI2 & $\mathrm{ABCC} 8$ & \multirow[t]{2}{*}{ Sulfonylureas } \\
\hline MODYI3 & $\mathrm{KCNJII}$ & \\
\hline MODYIO & INS & Insulin \\
\hline MODY6 & NEURODI & \multirow{7}{*}{$\begin{array}{l}\text { No specific treatment; oral antidiabetic } \\
\text { agents or insulin can be used based on } \\
\text { blood glucose levels }\end{array}$} \\
\hline MODY4 & IPFI & \\
\hline MODY8 & CEL & \\
\hline MODYII & BLK & \\
\hline MODY9 & PAX4 & \\
\hline MODY7 & KLFII & \\
\hline MODYI4 & APPLI & \\
\hline
\end{tabular}

Notes: Data from Aarthy et al, $2020 .^{15} *$ The pathogenicity of many of the rarer subtypes is disputed.

Abbreviations: MODY, maturity-onset diabetes of the young; HNF, hepatocyte nuclear factor; GCK, glucokinase; ABCC8, ATP binding cassette subfamily C member 8; KCNJII, potassium inwardly rectifying channel subfamily | member II; INS, insulin gene; NEURODI, neurogenic differentiation factor; IPF, insulin promoter factor; CEL, carboxyl ester lipase; BLK, B lymphocyte kinase; PAX4, paired box 4; KLFI I, Krueppel-like factor II; APPLI, adaptor protein, phosphotyrosine interacting with $\mathrm{PH}$ domain and leucine zipper I. 
parts of the world. Indeed, many forms of "MODY' that were earlier described and named in the literature have been shown to be nonpathogenic variants on further detailed analysis. The rarer varieties of MODY have recently been reviewed, and the implications of the diagnosis are listed in Table $3 .{ }^{15}$

\section{Challenges in the Management of Diabetes}

The ultimate aim of precision medicine is the development of a personalized treatment plan for each patient, that maximises the benefits of therapy while minimizing adverse effects so as to ensure optimal treatment outcomes. The application of a personalized approach to management starts even before the initiation of medications is considered, as there are often wide differences in the response among patients to lifestyle modification, which forms the cornerstone of diabetes management. For instance, it has been shown that Asian Indians need to perform far more intense physical activity than White Caucasians, in order to obtain equivalent cardiometabolic benefit. $^{16}$ A personalized approach is also ideal while prescribing diet to the patient with diabetes. Unfortunately, there is a scarcity of trained professionals capable of prescribing personalized lifestyle changes to people with diabetes, particularly in developing countries.

While beta-cell failure is the final common pathway to the development of all forms of diabetes, ${ }^{17-19}$ the exact mechanisms by which the beta cell damage occurs vary subtly, even among individuals who have been classified as having T2D. These mechanisms have been comprehensively described under the umbrella term, the "ominous octet". ${ }^{20}$ Knowledge of pathophysiologic mechanisms predominating in a given patient with T2D will help in deciding the most appropriate therapy for that particular patient. There are now more than 8 classes of anti-diabetic drugs available for the treatment of non-type 1 diabetes, each of which works on a different aspect of the pathophysiology of the disease. While all these drugs have been shown to be highly effective in controlling hyperglycemia especially in the context of clinical trials, it is often noted that their real-world efficacy is much lower. ${ }^{21}$ While most of this discrepancy can be explained by factors such as non-adherence to the treatment regimen, there is also a possibility that it may reflect differences in the pathophysiological basis of diabetes among individual patients, such that an individual patient responds better to one agent but not to another. The susceptibility to side effects of antidiabetic agents also varies considerably between patients.

While treatment options are usually straightforward in the case of monogenic diabetes (oral sulfonylureas for neonatal diabetes and MODY1 and 3, no treatment needed for MODY2), the situation is more complicated in the case of T2D where a myriad of pathophysiologies are at play. With this in mind, attempts have been made to see whether certain subgroups of patients with T2D respond better to certain classes of antidiabetic agents than others. Current knowledge of the pathophysiology of T2D tells us that Asian Indians tend to have more severe insulin deficiency (beta-cell defect) compared to white Caucasians, in whom insulin resistance appears to be the predominant defect. ${ }^{22}$ Asian Indians can therefore be expected to respond better to insulin-providing therapies early in the course of the disease, rather than to metformin, which is widely accepted as the first-line pharmacotherapy for T2D worldwide. Unfortunately, while this hypothesis is attractive, there are little real-world or clinical trial data to support it and this forms one of the main lacunae in the adoption of the personalized approach to diabetes care.

Nonetheless, attempts have been made recently to assess the differential response of individuals belonging to different ethnic groups, to various classes of antidiabetic agents. Gan et al recently attempted to assess the impact of ethnicity of the efficacy of three classes of antidiabetic agents viz. DPP-4 inhibitors, SGLT2 inhibitors and GLP1 receptor agonists. ${ }^{23}$ Their meta-analysis included 64 studies and showed that compared to individuals of white ethnicity, those of Asian ethnicity responded better to SGLT2 inhibitors and to some extent, DPP-4 inhibitors. However, it could not be determined from this study whether responses differed between East Asians and South Asians. Larger studies looking at individual-level variability in drug response are needed before the findings of this meta-analysis can be adopted into precision diabetes care. Also, more data are needed on the differential response of older diabetes medications such as metformin, sulfonylureas and thiazolidinediones which continue to be widely used worldwide on account of lower cost.

Efforts have also been made to understand the basis for individual variation in the risk of various side-effects due to antidiabetic agents. In particular, studies have identified variants of the organic cation transporter-1 (OCT1) as responsible for much of the gastrointestinal side effects experienced with metformin, as well as its efficacy. ${ }^{24}$ There is also some evidence that the side effects of 
thiazolidinediones (such as weight gain) are influenced by genetic variation. However, much more work needs to be done before these initial results can be translated into clinical practice.

Many categories of antidiabetic agents have been shown to disproportionately benefit certain patient subgroups. For instance, the American Diabetes Association now recommends the use of GLP-1 receptor agonists and SGLT2 inhibitors as second-line agents after metformin in individuals with T2D who also have cardiovascular or renal disease. ${ }^{25}$ However, a major hurdle in putting this recommendation into practice, ie, in transforming precision diabetes care to personalized diabetes care, is the cost of treatment. Often, it is found that the most appropriate treatment in the physiological sense is not the ideal treatment in the practical sense, simply because the patient cannot afford it. Efforts need to be put in place to ensure easy availability and affordability of these modern drugs to those patients who stand to benefit the most from it.

\section{Conclusions}

Personalised diabetes management is a powerful tool in the battle against diabetes, as it provides patients with the most effective and appropriate diagnostic, therapeutic and preventive modalities for their condition, thereby enabling them to achieve the best possible outcomes with the lowest incidence of side-effects. However, the field of personalized medicine in general, and personalized diabetes in particular, is still in its infancy, with various challenges that need to be addressed before this approach can be universally applied. While some of these challenges pertain to lack of data on the nature of diabetes in various populations, others are linked to inequities in the availability and affordability of various diagnostic and therapeutic modalities. It is to be hoped that further advances in this field will bring personalized care within the reach of every individual with diabetes.

\section{Disclosure}

The authors report no conflicts of interest in this work.

\section{References}

1. Parivallal T.Diabetes in Ancient India. In: Type 2 Diabetes in South Asians: Epidemiology, Risk Factors and Prevention. Mohan V, Rao GHR,editors. Under the Aegis of SASAT. Jaypee Brothers Medical Publishers; 2006:97-103.

2. Himsworth HP. Diabetes mellitus: its differentiation into insulin-sensitive and insulin-insensitive types. The Lancet. 1936;227 (5864):127-130. doi:10.1016/S0140-6736(01)36134-2
3. Chung WK, Erion K, Florez JC, et al. Precision Medicine in Diabetes: a Consensus Report From the American Diabetes Association (ADA) and the European Association for the Study of Diabetes (EASD). Diabetes Care. 2020; 43: 1617-1635.

4. Mohan V, Unnikrishnan R. Precision diabetes: where do we stand today? Indian J Med Res. 2018;148(5):472-475. doi:10.4103/ijmr. IJMR_1628_18

5. Ahlqvist E, Storm P, Käräjämäki A, et al. Novel subgroups of adult-onset diabetes and their association with outcomes: a data-driven cluster analysis of six variables. Lancet Diabetes Endocrinol. 2018;6(5):361-369. doi:10.1016/S2213-8587(18)30051-2

6. Zou X, Zhou X, Zhu Z, Ji L. Novel subgroups of patients with adult-onset diabetes in Chinese and US populations. Lancet Diabetes Endocrinol. 2019;7:9-11. doi:10.1016/S2213-8587(18) 30316-4

7. Bello-Chavolla OY, Bahena-López JP, Vargas-Vázquez A, et al. Metabolic syndrome study group; group of study CAIPaDi. CLINICAL characterization of data-driven diabetes subgroups in Mexicans using a reproducible machine learning approach. $B M J$ Open Diabetes Res Care. 2020;8:e001550.

8. Zaharia OP, Strassburger K, Strom A, et al. German Diabetes Study Group. Risk of diabetes-associated diseases in subgroups of patients with recent-onset diabetes: a 5-year follow-up study. Lancet Diabetes Endocrinol. 2019;7(9):684-694. doi:10.1016/S2213-8587(19)30187-1

9. Anjana RM, Baskar V, Nair ATN, et al. Novel subgroups of type 2 diabetes and their association with microvascular outcomes in an Asian Indian population: a data-driven cluster analysis: the INSPIRED study. BMJ Open Diabetes Res Care. 2020;8:e01506. doi:10.1136/bmjdrc-2020-001506

10. Dennis JM, Shields BM, Henley WE, Jones AG, Hattersley AT. Disease progression and treatment response in data-driven subgroups of type 2 diabetes compared with models based on simple clinical features: an analysis using clinical trial data. Lancet Diabetes Endocrinol. 2019;7:442-451. doi:10.1016/S2213-8587(19)30087-7

11. Dhanwal DK, Agarwal S, Garg S, Agarwal P. Clinical \& immunological profile of newly diagnosed patients with youth onset diabetes mellitus. Indian J Med Res. 2014;140:356-360.

12. Tattersall RB, Fajans SS. A difference between the inheritance of classical juvenile-onset and maturity-onset type diabetes of young people. Diabetes. 1975;24:44-53. doi:10.2337/diab.24.1.44

13. Murphy R, Ellard S, Hattersley AT. Clinical implications of a molecular genetic classification of monogenic beta-cell diabetes. Nat Clin Pract Endocrinol Metab. 2008;4:200-213. doi:10.1038/ ncpendmet 0778

14. Ellard S, Colclough K, Patel KA, Hattersley AT. Prediction algorithms: pitfalls in interpreting genetic variants of autosomal dominant monogenic diabetes. J Clin Invest. 2020;130:14-16. doi:10.1172/ JCI133516

15. Aarthy R, Aston-Mourney K, Mikocka-Walus A, et al. Clinical features, complications and treatment of rarer forms of maturity-onset diabetes of the young (MODY) - a review. $J$ Diabetes Complications. 2021;35:107640. doi:10.1016/j. jdiacomp.2020.107640

16. Iliodromiti S, Ghouri N, Celis-Morales CA, Sattar N, Lumsden MA, Gill JM. Should physical activity recommendations for South Asian adults be ethnicity-specific? Evidence from a cross-sectional study of South Asian and White European Men and Women. PLoS One. 2016;11:e0160024. doi:10.1371/journal.pone.0160024

17. Schwartz SS, Epstein S, Corkey BE, Grant SF, Gavin JR 3rd. Aguilar RB the Time Is Right for a New Classification System for Diabetes: Rationale and Implications of the B-Cell-Centric Classification Schema Diabetes Care. 2016;39:179-186.

18. Saisho Y. Changing the concept of Type 2 diabetes: beta cell workload hypothesis revisited. Endocr Metab Immune Disord Drug Targets. 2019;19:121-127. doi:10.2174/187153031866618082116 1825 
19. Saisho Y. An emerging new concept for the management of type 2 diabetes with a paradigm shift from the glucose-centric to beta cell-centric concept of diabetes - an Asian perspective. Expert Opin Pharmacother. 2020;21(13):1565-1578. doi:10.1080/14656566. 2020.1776262

20. DeFronzo, From the triumvirate to the ominous octet (Banting Lecture), Diabetes, 2009

21. Edelman SV, Polonsky WH. Type 2 diabetes in the real world: the elusive nature of glycemic control. Diabetes Care. 2017;40 (11):1425-1432. doi:10.2337/dc16-1974

22. Narayan KMV, Kanaya AM. Why are South Asians prone to type 2 diabetes? A hypothesis based on underexplored pathways. Diabetologia. 2020;63(6):1103-1109. doi:10.1007/s00125-020-05132-5
23. Gan S, Dawed AY, Donnelly LA, et al. Efficacy of Modern Diabetes Treatments DPP-4i, SGLT-2i, and GLP-1RA in white and Asian patients with diabetes: a systematic review and meta-analysis of randomized controlled trials. Diabetes Care. 2020;43(8):1948-1957. doi: $10.2337 / \mathrm{dc} 19-2419$

24. Florez JC. The pharmacogenetics of metformin. Diabetologia. 2017;60(9):1648-1655. doi:10.1007/s00125-017-4335-y

25. American Diabetes Association 9. Pharmacologic approaches to glycemic treatment: standards of medical care in diabetes-2021. Diabetes Care. 2021;44(Supplement 1):S111-S124. doi:10.2337/ dc21-S009

\section{Publish your work in this journal}

Pharmacogenomics and Personalized Medicine is an international, peer-reviewed, open access journal characterizing the influence of genotype on pharmacology leading to the development of personalized treatment programs and individualized drug selection for improved safety, efficacy and sustainability. This journal is indexed on the American Chemical Society's Chemical Abstracts Service (CAS). The manuscript management system is completely online and includes a very quick and fair peer-review system, which is all easy to use. Visit http://www.dovepress.com/testimonials.php to read real quotes from published authors. 